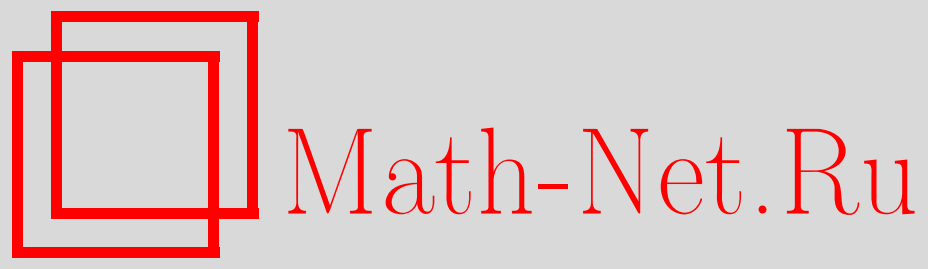

Э. В. Киссин, В. С. Шульман, Представления нильпотентных групп: расширения, нейтральные когомологии, пространства Понтрягина, Функи. анализ и его прил., 2015, том 49, выпуск 3, 79-83

DOI: https://doi.org/10.4213/faa3208

Использование Общероссийского математического портала MathNet.Ru подразумевает, что вы прочитали и согласны с пользовательским соглашением http://www. mathnet.ru/rus/agreement

Параметры загрузки:

IP: 54.196 .121 .252

26 апреля 2023 г., 14:39:05

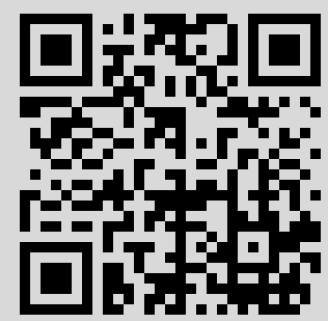


В заключение автор выражает глубокую благодарность рецензенту за ценные замечания.

\title{
ЛитерАТУРА
}

[1] В. П. Паламодов, в кн.: Итоги науки и техники, Современные проблемы математики, Фундаментальные направления, т. 72, ВИНИТИ, М., 1991, 5-134. [2] В. В. Лебедев, Функц. анализ и его прил., 47:1 (2013), 33-46. [3] E. M. Stein, Harmonic Analysis: Real-Valued Methods, Orthogonality and Oscillatory Integrals, Princeton University Press, Princeton, NJ, 1993. [4] И. М. Виноградов, Метод тригонометрических сумм в теории чисел, Наука, M., 1980. [5] Hua Loo-keng, Acta Sci. Sinica, 1:1 (1952), 1-76. [6] Г. И. Архипов, А. А. Карацуба, В. Н. Чубариков, Изв. АН СССР, сер. матем., 43:5 (1979), 971-1003. [7] И. А. Икромов, Матем. заметки, 87:5 (2010), 734755. [8] L. Erdös, M. Salmhofer, Math. Z., 257:2 (2007), 261-294. [9] В. И. Арнольд, А. Н. Варченко, С. М. Гусейн-заде, Особенности диффернцируемых отображений, ч. 1. Классификация критических точек каустик и волновых фронтов, Наука, М., 1982. [10] B. Randol, Trans. Amer. Math. Soc., 139 (1969), 279-285. [11] I. Svensson, Ark. Mat., 9:1 (1970), 11-22. [12] И. А. Икромов, Функц. анализ и его прил., 29:3 (1995), 16-24.

Самаркандский государственный университет e-mail: ikromov1@rambler.ru

Поступила в редакцию 18 августа 2013 г.

УДК 512.5

\section{Представления нильпотентных групп: расширения, нейтральные когомологии, пространства Понтрягина}

\author{
(с) 2015. Э. В. КисСин, В. С. ШульмАн
}

1. Введение. Неприводимые унитарные представления нильпотентных групп привлекали внимание многих специалистов. Известно, что они либо одномерны, либо бесконечномерны; для нильпотентных групп Ли Кирилловым [3] разработан метод орбит, позволяющий во многих случай получать конкретную и обозримую классификацию. Произвольные унитарные представления единственным образом раскладываются в прямые интегралы неприводимых.

В случае несимметричных представлений ситуация значительно менее определенная. По-прежнему неприводимые конечномерные представления одномерны (теорема Ли-Колчина [4]), но разложить произвольные представления в сумму неприводимых уже нельзя. Их можно раскладывать в сумму неразложимых представлений, но классификация последних обычно не представляется возможной.

Имеется естественная ситуация, в которой приходится изучать некоторую смесь конечномерных и унитарных представлений группы, - теория представлений групп в пространствах Понтрягина $\Pi_{k}$. Как показал Наймарк [5], J-симметричное представление связной нильпотентной группы в $\Pi_{k}$ имеет инвариантное подпространство размерности $k$; это позволяет рассматривать такие представления как специальные конструкции «симметризованных расширений» конечномерных представлений с помощью унитарных. Хотя проблема с конечномерной частью остается, она облегчается тем, что размерности возникающих 
конечномерных компонент ограничены индексом индефинитности. В данной работе последовательно рассматриваются 1) условия разложимости представлений нильпотентных групп; 2) разложимость расширений конечномерных представлений с помощью унитарных и 3$)$ строение $\Pi_{k}$-представлений.

2. Когомологии и расширения. Пусть $\lambda$ и $U-$ слабо непрерывные представления группы $G$ в гильбертовых пространствах $L$ и $\mathfrak{H}$ соответственно. Обозначим через $B(\mathfrak{H}, L)$ пространство ограниченных линейных операторов из $\mathfrak{H}$ в $L$, рассматриваемое как $G$-бимодуль. Когомологии группы с коэффициентами в $B(\mathfrak{H}, L)$ определяются стандартным образом (классический случай, чаще всего рассматриваемый в литературе, соответствует выбору в качестве $\lambda$ тривиального представления $\iota$ в одномерном пространстве). В частности, 1-коцикл - это слабо непрерывная функция $\xi: G \rightarrow B(\mathfrak{H}, L)$, удовлетворяющая условию

$$
\xi(g h)=\lambda(g) \xi(h)+\xi(g) U(h) \quad \text { для любых } g, h \in G .
$$

Условие тривиальности 1-когомологий особенно важно для теории представлений, поскольку оно обеспечивает тривиальность расширений. Напомним, что расширением представления $\lambda$ с помощью $U$ называется любое представление, имеющее замкнутое инвариантное подпространство $L$, сужение на которое эквивалентно $\lambda$, а представление, индуцированное в факторпространстве, эквивалентно $U$. Расширение считается тривиальным, если $L$ имеет инвариантное дополнение.

Стандартная конструкция расширений такова. Положим $\mathfrak{Z}=L \oplus \mathfrak{H}$. С каждым $(\lambda, U)$-коциклом $\xi$ можно связать представление $\pi$ на $\mathfrak{Z}$, действующее по правилу

$$
\pi(g)=\left(\begin{array}{cc}
\lambda(g) & \xi(g) \\
0 & U(g)
\end{array}\right) \quad \text { для } g \in G .
$$

Оно является расширением представления $\lambda$ с помощью $U$ и обозначается через $\mathfrak{e}(\lambda, U, \xi)$. Для того чтобы расширение $\mathfrak{e}(\lambda, U, \xi)$ было тривиальным, необходимо и достаточно, чтобы коцикл $\xi$ был кограницей.

Для $h \in G$ определим отображение $\operatorname{ad}_{h}: G \rightarrow G$ формулой

$$
\operatorname{ad}_{h}(g)=g h g^{-1} h^{-1} \quad \text { для всех } g \in G .
$$

Элемент $h$ называется энгелевым, если для любого $g \in G$ найдется такой номер $n=n_{g}$, что $\operatorname{ad}_{h}^{n}(g)=e$.

Теорема 1. Пусть $\lambda$ и $U-$ представления группъ $G$. Если

$$
\operatorname{Sp}(\lambda(h)) \cap \operatorname{Sp}(U(h))=\varnothing
$$

для некоторого энгелева элемента $h \in G$, mо $\mathscr{H}^{1}(\lambda, U)=0$.

Так как в нильпотентной группе все элементы энгелевы, то получаем следствие, играющее важную роль во всей работе:

Следствие 2. Пусть $\lambda u U-$ представления связной нильпотентной группзи $G$. Если $\operatorname{Sp}(\lambda(h)) \cap \operatorname{Sp}(U(h))=\varnothing$ для некоторого $h \in G$, то $\mathscr{H}^{1}(\lambda, U)=0$.

Следствие 3. Пусть $\pi$ - представление связной нильпотентной группь в банаховом пространстве $X$, и пусть $L$ - замкнутое инвариантное подпространство для $\pi$. Пусть $\lambda=\left.\pi\right|_{L}$, а $U$ - представление, индуцируемое в $X / L$. Если для некоторого $h \in G$ выполнено условие (1), то $Y$ имеет инвариантное дополнение в $X$.

Легко видеть, что этот результат не переносится на разрешимые группы. 
Пусть $\chi$ - характер группы $G$ (т. е. мультипликативный функционал на $G$ ). Будем говорить, что $\chi$ присоединен к представлению $\lambda$, если $\lambda(g) x=\chi(g) x$ для некоторого вектора $x \neq 0$ и всех $g \in G$. Множество всех характеров, присоединенных к $\lambda$, обозначим через $\operatorname{sign}(\lambda)$. Будем называть представление $\lambda$ моноmетическим, если $\operatorname{sign}(\lambda)$ одноточечное. Следующий (безусловно, известный, но неизвестно где опубликованный) результат немедленно вытекает из теоремы Ли и следствия 3.

Следствие 4. Всякое конечномерное представление связной нильпотентной группы является прямой суммой монотетичных представлений.

Везде ниже группа $G$ предполагается связной и нильпотентной.

Пусть $\lambda$ - представление группы $G$ в конечномерном пространстве $L, U-$ ее унитарное представление в гильбертовом пространстве $\mathfrak{H}$, а $\xi$ - некоторый $(\lambda, U)$-коцикл.

Теорема 5. Пусть $\pi=\mathfrak{e}(\lambda, U, \xi)$ - представление группъ $G$ в $\mathfrak{Z}=L \dot{+} \mathfrak{H}$, причем $\operatorname{dim} \mathfrak{H}=\infty$.

Существуют замкнутые $\pi$-инвариантные подпространства $\left\{X_{n}\right\}_{n=1}^{\infty}$, $\left\{Y_{n}\right\}_{n=1}^{\infty}$, maкue, чmo

$$
\mathfrak{Z}=X_{n}+Y_{n}, \quad X_{n+1} \subset X_{n}, Y_{n} \subset Y_{n+1} \text { для любого } n,
$$

все представления $\left.\pi\right|_{Y_{n}}$ подобны унитарным, кажсде подпространство $X_{n}$ содержит $L$, причем пересечение всех $X_{n}$ конечномерно, а замыкание объединения всех $Y_{n}$ имеет конечную коразмерность в $\mathfrak{Z}$.

Следствие 6. Если представление $\mathfrak{e}(\lambda, U, \xi)$ не раскладывается в прямую сумму двух подпредставлений, то $\operatorname{dim} \mathfrak{H}<\infty, \operatorname{sign}(\lambda)=\{\chi\}$ u $U=\chi \mathbf{1}_{\mathfrak{H}}$.

Изучая представления групп в пространствах с индефинитной метрикой, Исмагилов [1] ввел в рассмотрение специальный класс коциклов, к рассмотрению которого мы сейчас переходим. Обозначим через $\lambda^{\sharp}$ представление, сопряженное к $\lambda: \lambda^{\sharp}(g)=\lambda\left(g^{-1}\right)^{*}$. Пусть $C(\mathfrak{H}, L)$ - пространство непрерывных отображений из $G$ в $B(\mathfrak{H}, L)$. Для любого $c \in C(\mathfrak{H}, L)$ определим отображение $c^{\sharp}(g) \in C(L, \mathfrak{H})$, полагая $c^{\sharp}(g)=c\left(g^{-1}\right)^{*}$.

Ясно, что если $\xi$ есть $(\lambda, U)$-коцикл, то $\xi^{\sharp}$ есть $\left(U, \lambda^{\sharp}\right)$-коцикл, а отображение $\xi \xi^{\sharp}: G \times G \rightarrow B(L)$, определенное равенством $\xi \xi^{\sharp}(g, h)=\xi(g) \xi^{\sharp}(h)$, является $\left(\lambda, \lambda^{\sharp}\right)$-2-коциклом. Будем называть коцикл $\xi$ нейтральным, если $\xi \xi^{\sharp}$ - кограница, т. е. если существует такое отображение $\gamma \in C(L, L)($ nредцепь коцикла $\xi)$, чTо

$$
\xi(g) \xi^{\sharp}(h)=\left(d_{\lambda, \lambda}^{1} \gamma\right)(g, h)=\lambda(g) \gamma(h)-\gamma(g h)+\gamma(g) \lambda^{\sharp}(h) .
$$

Множество всех нейтральных коциклов обозначим через $\mathscr{Z}_{\nu}^{1}(\lambda, U)$. Можно показать, что коцикл, когомологичный нейтральному, нейтрален; поэтому естественно определяется множество $\mathscr{H}_{\nu}^{1}(\lambda, U)$ нейтральных 1-когомологий. Оно не всегда является подгруппой в $\mathscr{H}^{1}(\lambda, U)$ и может быть весьма сложно устроено даже в случае, когда $\lambda=\iota$, а $U=\iota_{m}$, представление единичными операторами в $\mathbb{C}^{m}$. Так, для группы $G=T_{n}$ верхнетреугольных $n \times n$-матриц с единицами на диагонали точное описание нейтральных $1-\left(\iota, \iota_{m}\right)$-когомологий (= коциклов), выглядит следующим образом.

Пусть $A$ - такая $m \times(n-1)$-матрица, что для $B=A^{*} A=\left(b_{i j}\right)$ выполнены условия

$$
\operatorname{Im} b_{i j}=0 \quad \text { при }|i-j|>1 \text {; }
$$


тогда $\xi(g)=A\left(g_{12}, \ldots, g_{n-1, n}\right)^{T}$ - нейтральный $\left(\iota, \iota_{m}\right)$-коцикл и все нейтральные коциклы имеют такой вид.

Следующий важный для дальнейшего результат утверждает, что для широкого класса представлений множество $\mathscr{H}_{\nu}^{1}(\lambda, U)$ является массивным.

Теорема 7. Пусть $G-$ связная локально компактная нильпотентная группа, а $U$ - ее унитарное представление, слабо содержащее, но не содер-

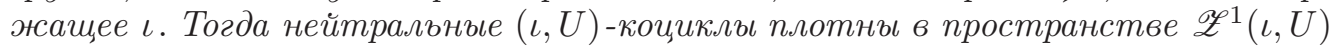
всех 1 -коииклов относительно топологии равномерной сходимости на компак$\max$.

3. $\boldsymbol{J}$-унитарные представления в $\boldsymbol{\Pi}_{\boldsymbol{k}}$. Напомним, что пространство Понтрягина $\Pi_{k}$ - это пространство $H$ с индефинитным скалярным произведением $[\cdot, \cdot]$, допускающее разложение в прямую сумму отрицательного подпространства размерности $k$ и подпространства, являющегося гильбертовым пространством относительно $[\cdot, \cdot]$. Оператор, действующий в $H$, называется $J$-унитарным, если он обратим и сохраняет $[\cdot, \cdot] . J$-унитарное представление группы $G$ в $H$ - это гомоморфизм группы $G$ в группу всех $J$-унитарных операторов.

По уже упоминавшейся во введении теореме М. А. Наймарка всякое J-унитарное представление связной нильпотентной группы в $\Pi_{k}$ имеет неположительное инвариантное подпространство размерности $k$. Если это подпространство отрицательно, то представление подобно унитарному. Поэтому интерес представляют лишь те представления, которые имеют инвариантные нейтральные (т. е. состоящие из таких векторов $x$, что $[x, x]=0$ ) подпространства.

В дальнейшем через $L$ обозначается максимальное инвариантное нейтральное подпространство представления $\pi$. Его $J$-ортогональное дополнение $L^{[\perp]}$ также инвариантно, однако разложение $H=L[+] L^{[\perp]}$ уже неверно, более того, $L \subset L^{[\perp]}$. Мы выбираем замкнутое подпространство $\mathfrak{H} \subset L^{[\perp]}$, дополнительное к $L$, и нейтральное подпространсто $M \subset H$, дополнительное к $L^{[\perp]}$; тогда блок-матричная запись представления относительно разложения $H=L \oplus \mathfrak{H} \oplus M$ имеет вид

$$
\pi=\left(\begin{array}{ccc}
\lambda & \xi & \gamma \\
0 & U & \eta \\
0 & 0 & \mu
\end{array}\right)
$$

Здесь представление $U$ в $\mathfrak{H}$ подобно унитарному (и становится унитарным после изменения скалярного произведения на $\mathfrak{H}), \xi-$ это $(\lambda, U)$-коцикл, $\mu(g)=$ $\lambda\left(g^{-1}\right)^{*}, \eta(g)=\xi\left(g^{-1}\right)^{*}, \gamma(g)^{*}=\gamma\left(g^{-1}\right)$, причем

$$
\gamma(g h)=\lambda(g) \gamma(h)+\xi(g) \eta(h)+\gamma(g) \mu(h) .
$$

Мы видим, что коцикл $\xi$ нейтрален и $-\gamma-$ его предцепь. Обратно, имея нейтральный коцикл и выбирая предцепь, можно построить $J$-унитарное представление. Это позволяет применять результаты о расширениях в теории П ${ }_{k}$-представлений. Перечислим некоторые из этих применений. Напомним, что группа $G$ предполагается связной и нильпотентной.

Теорема 8. Пусть L - нейтральное инвариантное подпространство J-унитарного представления $\pi$. Существует убываяшая последовательность $H_{m}$ замкнутых инвариантных подпространств, содержащих L, такая, что все $H_{m}^{[\perp]}$ положительны $и \bigcap_{m} H_{m}$ конечномерно. 
Этот факт (аппроксимативная расщепляемость) можно рассматривать как вариант разложимости произвольного представления в «прямую сумму» последовательности унитарных представлений (в $E_{k}=H_{k-1} \ominus H_{k}$ ) и представления в конечномерном (возможно, вырожденном) подпространстве $\bigcap_{m} H_{m}$. Отметим, что для группы Лоренца подобный результат был получен в [2].

Представляет интерес и другой вариант разложимости (уже в конечную $J$-ортогональную прямую сумму). Будем называть представление в $\Pi_{k}$-пространстве $H$ П-разложимым, если $H=H_{1}[+] H_{2}$, где $H_{1}$ и $H_{2}$ инвариантны и не положительны. В противном случае скажем, что представление П-неразложимо. Ясно, что всякое представление является прямой суммой конечного числа П-неразложимых. Для представлений в конечномерных пространствах такое разложение единственно (с точностью до изоморфизма), а в общем случае ответ пока не известен. Вопрос о классификации конечномерных П-неразложимых представлений тоже пока далек от полного решения.

Назовем представление примарным, если его сужение на максимальное инвариантное нейтральное подпространство монотетично. Следующий результат является ответом на один из вопросов, которые привели к появлению данной работы.

Теорема 9. (i) Eсли группа $G$ коммутативна, то все ее П-неразложимые представления примарны.

(ii) Существует П-неразложимое представление группъ Гейзенберга $T_{3}$, не являющееся примарным.

\title{
ЛитерАТУРА
}

[1] Р. С. Исмагилов, Матем. заметки, 35:1 (99-105). [2] Р. С. Исмагилов, Изв. АН СССР, сер. матем., 30:3 (1966), 497-522. [3] А. А. Кириллов, Функц. анализ и его прил., 2:1 (1968), 96-98. [4] E. R. Kolchin, Ann. of Math., 49:1 (1948), 1-42. [5] М. А. Наймарк, Изв. АН СССР, сер. матем., 27:6 (1963), 1181-1185.

STORM, London Metropolitan University

e-mail: e.kissin@londonmet.ac.uk

Поступила в редакцию

Вологодский государственный университет

e-mail: shulman.victor80@gmail.com

\section{УДК 517.5}

\section{Неравенство Никольского и функциональные классы на компактных группах Ли*}

\author{
(c) 2015. Е. Д. Нурсултанов, М. В. Ружанский, С. Ю. Тихонов
}

1. Введение. Классическое неравенство Никольского для тригонометрических полиномов $T_{L}$ степени не выше, чем $L$, записывается следующим обра3ом [7]:

$$
\left\|T_{L}\right\|_{L^{q}(\mathbb{T})} \leqslant 2 L^{1 / p-1 / q}\left\|T_{L}\right\|_{L^{p}(\mathbb{T})}
$$

*Первый автор был поддержан грантами МОН РК 4080 ГФ-4, 3311 ГФ-4. Второй автор был поддержан EPSRC Leadership Fellowship, EPSRC грант EP/K039407/1, и Leverhulme Grant RPG-2014-02. Третий автор был поддержан грантами 2014-SGR-289 от AGAUR (Generalitat de Catalunya), МТМ2014-59174-Р и РФФИ-13-01-00043. 